\title{
Abstract from Current Literature
}

Effects of birth size, post-natal growth and current size on insulin resistance in 9-year-old children: a prospective cohort study

Melissa J. W, Michael J. D, Lynne C. G, Bianca L. D, Julie $\mathrm{A}$. $\mathrm{O}$ et al.

Discipline of Obstetrics \& Gynaecology, School of Paediatrics \& Reproductive Health, The University of Adelaide, Australia, Discipline of Public Health, School of Population Health, Australia, Research Centre for the Early Origins of Health and Disease, Robinson Institute, Australia, Department of Medical Statistics, Faculty of Epidemiology and Population Health, London School of Hygiene and Tropical Medicine, London, UK, Melissa J. Whitrow, Email: melissa.whitrow@adelaide.edu.au

The influence of pre-natal conditions on later type 2 diabetes risk factors such as insulin resistance (IR) may be mediated by post-natal growth trajectory. We aimed to investigate the association of body size at birth and 9 years with IR at 9 years. Using data from a prospective Australian cohort study, we examined the influence of body size from birth to 9 years [zscore for weight or body mass index (BMI)] on IR at 9 years (estimated by homeostasis model assessment). At age 9 years, 151 children provided a fasting blood sample. $z-B M l$ at age 9 was positively associated with IR. Birth z-BMI was inversely associated with IR only after adjustment for z-BMI at age 9 years. This may be interpreted as an effect of accelerated growth between birth and 9 years on IR. There was a statistically significant interaction between birth and 9-year z-BMI. Results from regression models including $z-B M l$ at all available time points (birth, 6 and 12 months, and 2, 3.5 and 9 years) indicate a possible inverse association between body size at 3.5 years and HOMA-IR at 9 years. Results were similar when the analyses were repeated with $z-$ weight substituted for z-BMI. These results add to the body of evidence concerning the importance of growth in early life for later IR, and highlight a possible interaction between pre- and post-natal growth. The potential influence of growth at around 3.5 years for HOMA-IR at 9 years warrants further investigation.

European Journal of Pediatrics@ Springer-Verlag Berlin Received: 3 December 2012, Revised: 18 April 2013, Accepted: 19 April 2013, Published online: 8 May 2013
Acute Kidney Injury in Asphyxiated Newborns Treated with Therapeutic Hypothermia

Selewski DT, Jordan BK, Askenazi DJ, Dechert RE, Sarkar S.

Objective: To test the hypothesis that acute kidney injury (AKI) would be independently associated with increased morbidity and mortality.

Study design: A total of 96 consecutively cooled infants were reviewed retrospectively. Modified Acute Kidney Injury Network criteria were used to classify AKI based on absolute rise in serum creatinine ( $\mathrm{SCr}$ ) level from a previous trough (stage $\mathrm{I}$, rise in $\mathrm{SCr}$ of $0.3 \mathrm{mg} / \mathrm{dL}$ or $\mathrm{SCr} 150-<200 \%$; stage II, rise in SCr of $200-<300 \%$; stage III, rise in SCr of e" $300 \%$, SCr $2.5 \mathrm{mg} / \mathrm{dL}$, or dialysis). Outcomes were mortality, duration of neonatal intensive care unit (NICU) stay, and duration of mechanical ventilation.

Results: AKI occurred in 36 of 96 infants (38\%). Overall mortality was $7 \%$ and was higher for those with $\mathrm{AKI}$, with the difference approaching statistical significance $(14 \%$ vs $3 \%$ in those without $\mathrm{AKI}$; $P=.099)$. Patients with AKI stayed longer in the NICU (mean, $15.4 \pm 9.3$ days vs $11 \pm 5.9$ days; $P=.014$ ) and required prolonged mechanical ventilation (mean, $9.7 \pm 5.9$ days vs $4.8 \pm 3.7$ days; $P<.001)$. On multivariate analysis, AKI remained predictive of prolonged duration of mechanical ventilation and prolonged NICU stay.

Conclusion: We used the Acute Kidney Injury Network definition for AKI in asphyxiated newborns undergoing therapeutic hypothermia to demonstrate that the incidence of AKI remains high, but lower than rates published before the advent of therapeutic hypothermia. We highlight the importance of recognizing AKI in asphyxiated newborns undergoing therapeutic hypothermia, along with the potential benefits of early recognition.

The Journal of Pediatrics, Volume 162, Issue 4 , Pages 725-729.e1, April 2013 
A preliminary study of the variability in location of the ureteral orifices with bladder filling by fluoroscopic guidance: the gender difference

Hwang I, Kim S , Yu H, Hwang E, Jung S, Kang T et al.

Department of Urology, Chonnam National University Medical School, 8, Hak-dong, Dong-ku, Gwangju, 501-

757, South Korea, Sun-Ouck Kim, Email: seinsena@hanmail.net

Background and purpose: To determine landmarks for stent positioning in both ureteral orifices (UOs) and the gender differences in their location in men and women.

Patients and methods: The location of the $U O$ and the bladder neck (BN) was measured fluoroscopically by the intravesical distal location of an open-ended catheter marked with radiopaque materials. We compared the location in men $(n=12)$ and women $(n=12)$ with a full bladder (hydrostatic pressure of 50 $\mathrm{CmH}_{2} \mathrm{O}$ ) or an empty bladder.

Results: The mean distances from BN to UO in men and women were significantly different both in an empty bladder ( $2.5 \pm 0.4$ and $2.1 \pm 0.3 \mathrm{~cm}$, respectively) and in a full bladder $(2.9 \pm 1.0$ and $2.3 \pm 0.6 \mathrm{~cm}$, respectively). The location of UO was changed by bladder filling in women but not in men. In women, most UOs were found superior to the symphysis pubis (SP) in empty bladder (66.6 \%). Most of this location was observed at behind the upper boarder of SP in full bladder of women $(75 \%)$. The BN of women was located at the lower level in basal state compared to men. Also, the location of $\mathrm{BN}$ was markedly changed by bladder fulling in women $(p=0.04)$ but not in men.

Conclusions: Significant gender differences were observed in the location of UO and BN. Clinicians should keep in mind the anatomical differences between men and women during fluoroscopic-guided procedure.

Keywords: Ureteral stent, Ureteral orifice, Bladder neck, Fluoroscopy

International Urology and Nephrology@ Springer

Science + Business Media

Received: 24 February 2013, Accepted: 30 April 2013, Published online: 9 May 2013
International Urology and Nephrology $\odot$ Springer Science + Business Media

Exercise modality and metabolic efficiency in children

Bob G. F. Verweij ${ }^{1}$, Lee Stoner ${ }^{2}$ and Sarah P. Shultz ${ }^{2}$

${ }^{1}$ Department of Medicine, Utrecht University, Utrecht, Netherlands, ${ }^{2}$ School of Sport and Exercise, Massey University, PO Box 756, Wellington, 6140, New Zealand

Sarah P. Shultz, Email: S.P.Shultz@massey.ac.nz

Current exercise prescription guidelines for children recommend at least $60 \mathrm{~min}$ of moderate-to-vigorous physical activity every day. However, little is known about the efficacy of different cardiorespiratory exercise modalities prescribed to the pediatric cohort. Sixteen healthy children (8-12 years) completed 5-min trials of treadmill walking, cycling, and elliptical training in a randomized order. The treadmill walking speed was determined from measurements collected during a selfselected walking trial. The workloads for treadmill walking, cycling, and elliptical training were matched $(40.3 \mathrm{~W})$. Mechanical efficiency (ME\%), perceived exertion (RPE), oxygen uptake, metabolic equivalents, and net energy expenditure were measured. $\mathrm{ME} \%$ in walking was significantly higher than in cycling $(P=$ $0.001)$ and elliptical training $(P<0.001)$, and cycling was significantly higher than elliptical training $(P=$ $0.003)$. RPE in walking was significantly lower than in elliptical training $(P=0.006)$ but not from cycling ( $P$ $=0.314)$, and cycling resulted in significantly lower RPE than elliptical training $(P=0.021)$.

Conclusion: Treadmill walking appears to be the most efficacious exercise prescription for otherwise healthy children; however, longitudinal studies need to be implemented to investigate the long-term benefits of each exercise modality.

European Journal of Pediatrics@ Springer-Verlag Berlin Received: 24 February 2013, Revised:

22 April 2013, Accepted: 23 April 2013, Published online: 5 May 2013 